\title{
Atipias e lesões intraepiteliais cervicais: uma comparação entre pacientes da rede pública e privada de saúde
}

\author{
Atypias and cervical intraepithelial lesions: a comparison between pa- \\ tients in the public and private health network
}

Dyenifer Portela De Lima ${ }^{1}$ Denise Wohlmeister Paula WiethölteR ${ }^{1}$ IFaculdade Especializada na Área da Saúde do Rio Grande do Sul (FASURGS), Passo Fundo/RS
Resumo O câncer do colo do útero (CCU) é considerado um grande problema de saúde pública, sendo a segunda neoplasia mais frequente entre as mulheres, estando diretamente relacionado com a infecção pelo HPV. O objetivo do presente estudo foi comparar o diagnóstico de atipias e lesões intraepiteliais cervicais entre a rede pública e privada de saúde. Foram analisados 1.406 laudos (referentes a julho de 2015 a julho de 2019) e coletados dados referentes a idade, histórico de atipias/ lesões intraepiteliais cervicais, agentes inflamatórios responsáveis por alterações reativas (perfil epidemiológico) e grau das atipias (ASC-US e ASC-H) e lesões (HSIL, LSIL, carcinoma e AG), assim como se estes dados eram provenientes da rede pública ou privada de saúde. Não foram encontradas diferenças entre a rede pública e privada em relação ao diagnóstico de atipias. Para o diagnóstico de LSIL e AG um maior percentual foi encontrado na rede privada (respectivamente, $72,63 \%$ vs. $27,37 \%$; $=0,043$ e $100 \%$ vs. $0 \%$; $=0,039$ ). Enquanto para HSIL e carcinoma um maior percentual foi encontrado na rede pública (respectivamente, $56,79 \%$ vs. $43,21 \%$; $\mathrm{p}=0,041$ e $66,67 \%$ vs. $33,33 \%$; $\mathrm{p}=$ 0,037). Com base nos dados analisados, evidencia-se um maior percentual de lesões intraepiteliais cervicais de alto grau (HSIL) e carcinoma na rede pública em comparação com a rede privada, o que indica que essas pacientes podem estar expostas a um maior risco de desenvolvimento de CCU.

Palavras-chave: Citologia; Lesões intraepiteliais; Neoplasia; HPV; PREVENÇÃo.

Abstract Cervical cancer (CC) is considered a major public health problem, being the second most frequent neoplasm among women, being directly related to HPV infection. The aim of the present study was to compare the diagnosis of atypia and cervical intraepithelial lesions between the public and private health network. Were analyzed 1.406 reports (from July 2015 to July 2019) and collected data regarding age, history of atypia / cervical intraepithelial lesions, inflammatory agents responsible for reactive changes (epidemiological profile) and degree of atypia (ASC-US and ASC -H) and lesions (HSIL, LSIL, carcinoma and AG), as well as whether these data came from the public or private healthcare network. No differences were found between the public and private network in relation to the diagnosis of atypia. For the diagnosis of LSIL and AG, a higher percentage was found in the private 
network (respectively, $72,63 \%$ vs. $27,37 \%$; $p=0,043$ and $100 \%$ vs. $0 \%$; $p$ $=0,039$ ). While for HSIL and carcinoma a higher percentage was found in the public network (respectively, $56,79 \%$ vs. $43,21 \%$; $=0,041$ and $66,67 \%$ vs. $33,33 \% ; p=0,037)$. Based on the analyzed data, there is a higher percentage of high-grade cervical intraepithelial lesions (HSIL) and carcinoma in the public network compared to the private network, which indicates that these patients may be exposed to a higher risk of developing CC.

Keywords: Cytology; Intraepithelial Lesions; Neoplasm; HPV; Prevention.

\section{INTRODUÇÃo}

O câncer do colo do útero (CCU) representa mundialmente o segundo tipo de câncer mais frequente entre as mulheres, sendo uma neoplasia causada principalmente pela infecção do Papilomavírus Humano (HPV). Esse referido vírus ocasiona alterações no epitélio cervical, classificadas como lesões intraepiteliais cervicais, que podem ser de baixo ou alto grau, as quais podem evoluir e, quando não tratadas, desenvolver o CCU. ${ }^{1}$ No entanto, é considerada uma das poucas neoplasias que possui estratégias de prevenção primária e secundária, oferecidas inclusive pelo Sistema Único de Saúde (SUS), no Brasil.

Como prevenção primária, vacinas são disponibilizadas, visando proteger contra a infecção pelo HPV. A vacina quadrivalente, disponível no SUS e na rede privada, confere proteção aos subtipos de HPV de alto e baixo risco oncogênico, que são os subtipos 16 e 18, 6 e 11, respectivamente, reconhecidos como mais frequentes. ${ }^{2}$ Em 2017, a Agência Nacional de Vigilância Sanitária (Anvisa) registrou a vacina nonavalente, expandindo a prevenção para os subtipos de HPV 31, 33, 45, 52 e 58, além dos subtipos abrangidos pela vacina quadrivalente. ${ }^{3}$
Já a prevenção secundária tem como base a detecção precoce de lesões que possam evoluir para o $\mathrm{CCU}$, a qual é realizada pelo exame Preventivo do Câncer do Colo Uterino, também conhecido como Teste de Papanicolau. Sendo esse considerado um excelente método para o diagnóstico e o monitoramento das lesões intraepiteliais cervicais, causadas pelo $\mathrm{HPV}^{4} \mathrm{e}$ tido como um exame de fácil realização e de baixo custo.

No Brasil, a rede pública utiliza as Diretrizes Brasileiras para o Rastreamento do Câncer do Colo do Útero, que preconiza a realização do exame anualmente, em mulheres dos 25 aos 64 anos de idade, ou que já tenham vida sexual ativa. Após dois exames consecutivos com resultados negativos, é recomendado realizar novamente o exame apenas depois de três anos. ${ }^{5}$

Apesar da eficácia dos métodos profiláticos, ainda existem altos níveis de incidência do CCU. Segundo o Instituto Nacional do Câncer (INCA), em 2018 no Brasil, o número de casos estimado foi de 16.370, representando $8,1 \%$ do número total dos casos de câncer em mulheres. ${ }^{6} \mathrm{Na}$ América Latina a incidência chega a mais de 56 mil mulheres por ano. ${ }^{7}$

Ainda assim, a detecção precoce das lesões intraepiteliais cervicais vem contri- 
buindo para a redução de muitos casos de CCU. A importância do diagnóstico precoce está relacionada ao estágio em que as lesões se encontram, para que possam ser controladas e tratadas. Além disso, após o tratamento permanece a importância do monitoramento, para prevenir a recidiva das lesões. ${ }^{8}$

Com isso, faz-se necessário classificar cada lesão de acordo com o grau de evolução. O Sistema de Bethesda é utilizado mundialmente para padronizar os laudos de diagnóstico citológico. ${ }^{9}$ No Brasil, para os laudos da rede pública, se utiliza a Nomenclatura Brasileira para Laudos Cervicais e Condutas Preconizadas. ${ }^{10}$ Ambos classificam as células escamosas atípicas, quando detectadas. ${ }^{9-11}$ Para tanto, tem-se a seguinte classificação: células escamosas atípicas de significado indeterminado (atypical squamous cells of undetermined significance ASC-US) e células escamosas atípicas não sendo possível excluir lesão de alto grau (atypical squamous cells cannot exclude and HSIL - ASC-H). ${ }^{12}$

Além disso, também se tem a classificação de lesão intraepitelial escamosa de baixo grau (low-grade squamous intraepithelial lesion - LSIL) e lesão intraepitelial escamosa de alto grau (high-grade squamous intraepithelial lesion - HSIL). ${ }^{13} \mathrm{O}$ diagnóstico de carcinoma de células escamosas indica que as células infectadas pelo vírus penetraram a membrana basal, originando então o CCU, processo que pode demorar de 15 a 20 anos para se desenvolver. E de forma mais rara temos o diagnóstico de atipias em células glandulares $(\mathrm{AG}) .^{14}$
Atualmente, é possível observar que o risco para o desenvolvimento do CCU, pode estar relacionado a diversos fatores juntamente com a infecção pelo HPV. Com relação à idade das mulheres, sabe-se que mulheres em idade reprodutiva são as mais propensas a adquirir o HPV. Outros fatores também vêm sendo relacionados, como o sistema imunológico de cada paciente, a presença de infecções sexualmente transmissíveis (IST) e a multiplicidade de parceiros. ${ }^{15}$

$\mathrm{Na}$ rede pública, o exame Preventivo do Câncer do Colo Uterino, é realizado por apenas 15\% das mulheres brasileiras com mais de 20 anos, não sendo um número satisfatório. ${ }^{16}$ A carência no diagnóstico precoce das alterações citológicas prejudica o tratamento, pois muitas vezes as lesões são diagnosticadas em estágios mais avançados. Sendo então, de suma importância o diagnóstico precoce e o monitoramento das lesões. ${ }^{17}$

Dados do Ministério da Saúde demonstram que na região sul do país, dentre as mulheres que realizaram o exame Preventivo do Câncer do Colo Uterino no período de 2002 a 2003, em torno de $40 \%$ o realizaram na rede pública, e $60 \%$ na rede privada. ${ }^{18}$ Muller et al. (2008) apontam que no estado do Rio Grande do Sul 32,2\% das mulheres que realizaram o exame preventivo nos últimos anos foram atendidas pela rede pública de saúde, enquanto $67,8 \%$ realizaram o exame pela rede privada. ${ }^{19}$

Alguns estudos reportam a prevalência nos diferentes graus de lesões diagnosticados e sua relação com o desenvolvimento do 
$\mathrm{CCU}_{17-20}$, no entanto, ainda são escassos os relatos comparando rede pública e privada de saúde. Acredita-se que o tema da presente pesquisa venha preencher essa lacuna no conhecimento, contribuindo para o desenvolvimento de melhores políticas de prevenção e detecção precoce do CCU.

Desse modo o objetivo do presente estudo foi comparar o diagnóstico de atipias e lesões intraepiteliais cervicais entre a rede pública e privada.

\section{MAterial e métodos}

\section{Desenho do estudo}

Trata-se de um estudo observacional transversal retrospectivo, por meio da análise de laudos de exame Preventivo do Câncer do Colo Uterino, comparando as redes de saúde em que foram realizados, pública ou privada, em relação ao grau de atipias e lesões diagnosticadas.

O estudo foi realizado no Laboratório de Citopatologia Petry \& Wohlmeister Ltda., localizado na cidade de Carazinho/RS.

\section{Amostra do estudo}

A amostra abordada foi composta por mulheres que realizaram o exame Preventivo do Câncer do Colo Uterino, com diagnóstico citológico de atipia ou lesão intraepitelial. Os dados são referentes aos laudos preexistentes no banco de dados do laboratório em um período de quatro anos, de julho de 2015 a julho de 2019.

\section{Critérios de inclusão e exclusão}

Foram incluídos no estudo laudos de mulheres diagnosticadas com atipias ou lesões intraepiteliais. Sendo excluídos os laudos incompletos e com resultados de diagnósticos insatisfatórios para avaliação.

O banco de dados do laboratório foi analisado, por meio dos laudos citológicos. O perfil epidemiológico (idade, histórico de atipias/lesões intraepiteliais cervicais e agentes inflamatórios responsáveis por alterações reativas) e o grau das atipias (ASC-US e ASC-H) e lesões (LSIL, HSIL, Carcinoma e AG) diagnosticadas nas pacientes, foram comparados conforme a rede de saúde em que foram realizados, pública ou privada.

\section{Cálculo amostral}

O número amostral foi estabelecido pelo método de amostragem probabilística, com cálculo amostral ${ }^{21}$ considerando o número total de habitantes do sexo feminino no município do estudo (Carazinho/RS), de 31.037 mulheres, um grau de confiança correspondente a 95\% e erro amostral de $5 \%$ resultando em um número amostral de 350 laudos por ano. Os dados obtidos foram baseados nos resultados do Censo de 2010, realizado pelo Instituto Brasileiro de Geografia e Estatística. ${ }^{22}$

\section{Aspectos éticos}

Este projeto foi elaborado de acordo com a resolução 466/12 do Conselho Nacional de Saúde, relativa à pesquisa em seres humanos e aprovado com o Parecer Consubstanciado 
do Comitê de Ética em Pesquisa número 3.446.156.

\section{Análise estatística}

Os dados foram apresentados em número absoluto e percentual. O teste de Qui-quadrado, ou quando pertinente o teste exato de Fisher, foram utilizados para comparação entre grupos (rede pública vs. rede privada). Adotou-se um nível de significância de $\mathrm{p}<$ 0,05. Os dados foram tabulados no Software Microsoft Excel, e a análise estatística foi realizada no Software R versão 3.6.1.

\section{RESULTADOS}

Foram avaliados 1.406 laudos de exame Preventivo do Câncer do Colo Uterino, de pacientes diagnosticadas com atipias e lesões intraepiteliais cervicais. Verificou-se que $68,07 \%$ das pacientes abordadas no estudo foram atendidas pela rede privada de saúde $(\mathrm{n}=957)$ e $31,93 \%$ pela rede pública $(\mathrm{n}=$ 449). Como pode ser observado na Tabela 1 a maioria das pacientes se encontrava na faixa etária de 26 a 35 anos de idade (31,01\%), sem histórico de atipias ou de lesões intraepiteliais cervicais $(93,17 \%)$. Especificamente em relação as atipias, encontrou-se um maior percentual para ASC-US (46,59\%). Já para as lesões intraepiteliais cervicais uma maior frequência foi constatada para LSIL (41,32\%). Além disso, o agente inflamatório encontrado com maior frequência foi Gardnerella vaginalis (6,97\%).

Ao comparar os dados da rede pública e privada (Tabela 1) observou-se uma maior adesão pelo exame na rede privada, para as faixas etárias entre 15 e 75 anos $(p=0,031$ a 0,041$)$. Por outro lado, ao considerar pacientes com idade inferior a 15 anos, uma maior adesão pelo exame foi observada na rede pública $(\mathrm{p}=0,050)$.

Tanto em relação à presença de histórico de atipias e lesões intraepiteliais cervicais, quanto à ausência, observou-se um maior percentual na rede privada (respectivamente, $84,38 \%$ vs. $15,62 \%$; $\mathrm{p}=0,034$ e $66,87 \%$ vs. $33,13 \% ; p=0,034)$. Não foram constatadas diferenças significativas para os diagnósticos de atipias. Porém, para o diagnóstico de lesões intraepiteliais cervicais observou-se diferenças. Para o diagnóstico de LSIL e AG um maior percentual foi encontrado na rede privada (respectivamente, $72,63 \%$ vs. $27,37 \%$; $=0,043$ e $100 \%$ vs. $0 \%$; $p=0,039$ ). Enquanto para HSIL e carcinoma um maior percentual foi encontrado na rede pública (respectivamente, $56,79 \%$ vs. $43,21 \% ; \mathrm{p}=$ $0,041$ e $66,67 \%$ vs. $33,33 \%$; $=0,037)$. Em relação aos agentes inflamatórios responsáveis por alterações reativas nos laudos analisados, foram encontradas diferenças para Gardnerella vaginalis, na qual um maior percentual foi encontrado para a rede pública $(59,18 \%$ vs. 40,82\%; p = 0,041), já para Candida $s p p$ foi encontrado um maior percentual para a rede privada (78,95\% vs. $21,05 \%$; $\mathrm{p}=0,039)$.

Em adição, ao analisar a distribuição de atipias e lesões intraepiteliais cervicais por faixas etárias (Tabela 2) observou-se para o diagnóstico de ASC-US um maior percentual para a faixa etária de 26-35 anos (31\%) como também para ASC-H $(45,78 \%)$. Enquanto para o diagnóstico de LSIL observou-se um maior percentual para a faixa etária de 15-25 anos (37,69\%) e para a faixa etária de 26-35 anos $(27,19 \%)$. 
Tabela 1 - Perfil epidemiológico, atipias e lesões intraepiteliais cervicais de pacientes da rede privada e pública de saúde $(\mathrm{n}=1406)$.

\begin{tabular}{|c|c|c|c|c|}
\hline & $\begin{array}{c}\text { Total } \\
(\mathrm{n}=1406)\end{array}$ & $\begin{array}{c}\text { Rede } \\
\text { privada } \\
(\mathrm{n}=957)\end{array}$ & $\begin{array}{l}\text { Rede pública } \\
\qquad(\mathrm{n}=449)\end{array}$ & Valor-p \\
\hline \multicolumn{5}{|l|}{ Idade (anos) } \\
\hline$<15$ & $3(0,21 \%)$ & $1(33,33 \%)$ & $2(66,67 \%)$ & 0,029 \\
\hline $15-25$ & $416(29,59 \%)$ & $294(70,67 \%)$ & $122(29,33 \%)$ & 0,031 \\
\hline $26-35$ & $436(31,01 \%)$ & $318(72,94 \%)$ & $118(27,06 \%)$ & 0,033 \\
\hline $36-45$ & $256(18,21 \%)$ & $156(60,94 \%)$ & $100(39,06 \%)$ & 0,037 \\
\hline $46-55$ & $173(12,30 \%)$ & $117(67,63 \%)$ & $56(32,37 \%)$ & 0,036 \\
\hline $56-65$ & $92(6,54 \%)$ & $51(55,43 \%)$ & $41(44,57 \%)$ & 0,041 \\
\hline $66-75$ & $24(1,71 \%)$ & $17(70,83 \%)$ & $7(29,17 \%)$ & 0,034 \\
\hline$>75$ & $6(0,43 \%)$ & $3(50,00 \%)$ & $3(50,00 \%)$ & 0,05 \\
\hline \multicolumn{5}{|l|}{$\begin{array}{l}\text { Histórico de atipias/lesões } \\
\text { intraepiteliais cervicais }\end{array}$} \\
\hline Com histórico & $96(6,83 \%)$ & $81(84,38 \%)$ & $15(15,62 \%)$ & 0,034 \\
\hline Sem histórico & $1310(93,17 \%)$ & $876(66,87 \%)$ & $434(33,13 \%)$ & 0,034 \\
\hline \multicolumn{5}{|l|}{ Diagnóstico de atipias } \\
\hline ASC-US & $655(46,59 \%)$ & $447(68,24 \%)$ & $208(31,76 \%)$ & 0,052 \\
\hline ASC-H & $83(5,90 \%)$ & $49(59,04 \%)$ & $34(40,96 \%)$ & 0,051 \\
\hline \multicolumn{5}{|l|}{ Diagnóstico de lesões } \\
\hline \multicolumn{5}{|l|}{ epiteliais cervicais } \\
\hline LSIL & $581(41,32 \%)$ & $422(72,63 \%)$ & $159(27,37 \%)$ & 0,043 \\
\hline HSIL & $81(5,76 \%)$ & $35(43,21 \%)$ & $46(56,79 \%)$ & 0,041 \\
\hline Carcinoma & $3(0,21 \%)$ & $1(33,33 \%)$ & $2(66,67 \%)$ & 0,037 \\
\hline AG & $3(0,21 \%)$ & $3(100 \%)$ & $0(0 \%)$ & 0,039 \\
\hline \multicolumn{5}{|l|}{ Agentes inflamatórios } \\
\hline Gardnerella Vaginalis & $98(6,97 \%)$ & $40(40,82 \%)$ & $58(59,18 \%)$ & 0,041 \\
\hline Candida spp & $19(1,35 \%)$ & $15(78,95 \%)$ & $4(21,05 \%)$ & 0,039 \\
\hline Herpes & $1(0,07 \%)$ & $0(0 \%)$ & $1(100 \%)$ & 0,056 \\
\hline Trichomonas Vaginalis & $12(0,85 \%)$ & $1(8,33 \%)$ & $11(91,67 \%)$ & 0,054 \\
\hline
\end{tabular}

$\mathrm{p}<$ 0,05: diferença significativa. Dados apresentados em número absoluto (percentual). ASC-US = atypical squamous cells of undetermined significance; ASC-H = atypical squamous cells cannot exclude and HSIL; LSIL = low-grade squamous intraepithelial lesion; HSIL = high-grade squamous intraepithelial lesion; AG = atipias em células glandulares. 
Tabela 2 - Distribuição de atipias e lesões intraepiteliais cervicais por faixas etárias.

\begin{tabular}{|c|c|c|c|c|c|c|c|c|c|}
\hline \multirow{2}{*}{$\begin{array}{l}\text { Atipias/ } \\
\text { Lesões }\end{array}$} & \multicolumn{8}{|c|}{ Faixa etária (anos) } & \multirow{2}{*}{$\begin{array}{l}\text { Valor- } \\
\mathrm{p}\end{array}$} \\
\hline & $<15$ & $15-25$ & $26-35$ & $36-45$ & $46-55$ & $56-65$ & $66-75$ & $>75$ & \\
\hline \multirow[t]{2}{*}{ ASC-US } & 1 & 169 & 203 & 126 & 91 & 45 & 18 & 2 & 0,052 \\
\hline & $(0,15 \%)$ & $(25,80 \%)$ & $(31 \%)$ & $(19,24 \%)$ & $(13,90 \%)$ & $(6,87 \%)$ & $(2,75 \%)$ & $(0,30 \%)$ & \\
\hline \multirow[t]{2}{*}{ ASC-H } & 0 & 14 & 38 & 15 & 12 & 3 & 1 & 0 & 0,044 \\
\hline & $(0 \%)$ & $(16,87 \%)$ & $(45,78 \%)$ & $(18,07 \%)$ & $(14,46 \%)$ & $(3,61 \%)$ & $(1,20 \%)$ & $(0 \%)$ & \\
\hline \multirow[t]{2}{*}{ LSIL } & 2 & 219 & 158 & 97 & 63 & 36 & 5 & 1 & 0,041 \\
\hline & $(0,34 \%)$ & $(37,69 \%)$ & $(27,19 \%)$ & $(16,69 \%)$ & $(10,84 \%)$ & $(6,20 \%)$ & $(0,86 \%)$ & $(0,17 \%)$ & \\
\hline \multirow[t]{2}{*}{ HSIL } & 0 & 12 & 36 & 18 & 7 & 6 & 0 & 2 & 0,042 \\
\hline & $(0 \%)$ & $(14,81 \%)$ & $(44,44 \%)$ & $(22,22 \%)$ & $(8,64 \%)$ & $(7,41 \%)$ & $(0 \%)$ & $(2,47 \%)$ & \\
\hline \multirow[t]{2}{*}{ Carcinoma } & 0 & 0 & 1 & 0 & 0 & 1 & 0 & 1 & 0,046 \\
\hline & $(0 \%)$ & $(0 \%)$ & $(33,33 \%)$ & $(0 \%)$ & $(0 \%)$ & $(33,33 \%)$ & $(0 \%)$ & $(33,33 \%)$ & \\
\hline \multirow[t]{2}{*}{$\mathrm{AG}$} & 0 & 2 & 0 & 0 & 0 & 1 & 0 & 0 & 0,051 \\
\hline & $(0 \%)$ & $(66,67 \%)$ & (0\%) & $(0 \%)$ & $(0 \%)$ & $(33,33 \%)$ & $(0 \%)$ & $(0 \%)$ & \\
\hline
\end{tabular}

$\mathrm{p}<0$,05: diferença significativa. Dados apresentados em número absoluto (percentual). ASC-US = atypical squamous cells of undetermined significance;

ASC-H = atypical squamous cells cannot exclude and HSIL; LSIL = low-grade squamous intraepithelial lesion; $\mathrm{HSIL}=$ high-grade squamous intraepithelial lesion; $\mathrm{AG}=$ atipias em células glandulares .

\section{Discussão e CONCLUSÃo}

Os resultados obtidos revelam que a maior parte das pacientes tem entre 26 e 35 anos de idade e que apesar das diferentes formas de prevenção do CCU, ainda existe uma alta prevalência das lesões precursoras. Comparando as duas redes de atendimento, rede privada e rede pública, pode-se analisar que a diferença entre os diagnósticos encontrados não é significativa em relação às atipias, porém nas lesões de baixo e de alto grau, percebe-se uma diferença entre as duas redes de atendimento. A rede pública, em comparação com a rede privada, apresentou um percentual maior de lesões de alto grau (HSIL), o que indica que essas pacientes podem estar expostas a um maior risco de desenvolvimento de CCU. 
O SISCAN (Sistema de informação do câncer) é um programa de rastreamento do CCU que tem como objetivo a observação não apenas do exame realizado, mas da paciente como um todo. As lesões e atipias que são diagnosticadas devem seguir para acompanhamento da possível evolução dessas alterações e para tratamento quando necessário. Porém, o programa ainda apresenta limitações quanto as orientações à população-alvo e o correto seguimento após a realização do exame. ${ }^{23}$ Como consequência dessas limitações, observa-se o elevado número de lesões de alto grau na rede pública quando comparada com a rede privada em nosso estudo.

As alterações com maior percentual em nosso estudo foram ASC-US e LSIL, tanto na rede pública como na rede privada. Um estudo realizado por Trindade et al. ${ }^{24}$ demonstra que as alterações encontradas, representaram 2,7\% do total dos laudos analisados. Dessas alterações, 1,7\% são de ASC-US e $0,7 \%$ de LSIL, ${ }^{24}$ demonstrando, então, maior prevalência de ASC-US e de L-SIL, como relatado em nosso estudo.

Assim como Martins et al., ${ }^{25}$ que avaliaram no seu estudo mulheres com presença de alterações, $37,7 \%$ dos diagnósticos encontrados foram de LSIL e $18 \%$ ASC-US. ${ }^{25}$ Como observado também por Rocha et al. ${ }^{26}$ que identificaram uma prevalência de 48,4\% de ASC-US e 31,42\% de LSIL. Em geral, os relatos encontrados na literatura descrevem uma prevalência maior de ASC-US e de LSIL, corroborando com o presente estudo.

Já Costa e Barros, ${ }^{27}$ observaram em seu estudo uma prevalência do diagnóstico de LSIL de 26,7\% e 73,3\% para o diagnóstico de HSIL, em pacientes com diagnóstico ante- rior de ASC-US. Relatando então a evolução das alterações, de atipias ou lesões de baixo grau para lesões de alto grau. ${ }^{26-27}$

O diagnóstico de ASC-US apresentou em nosso estudo maior percentual na faixa etária dos 26-35 anos de idade, com 31\%. Freitas et al. ${ }^{29}$ explicam que a alta prevalência de ASC-US em mulheres jovens, frequentemente relatada, ocorre pelo fato de que elas encontram-se no período de idade reprodutiva, portanto, estão mais expostas a fatores de risco como as alterações inflamatórias, multiplicidade de parceiros e ISTs. ${ }^{29}$

As alterações inflamatórias com maior prevalência no estudo são causadas por Gardnerella vaginalis, 6,97\%. Assim como relatado por Fredrich e Renner, ${ }^{28}$ com 19,2\% de Gardnerella vaginalis, seguido de 1,57\% de Candida spp e $0,4 \%$ de Trichomonas vaginallis, apontados como responsáveis pelas inflamações detectadas. A presença de agentes inflamatórios causa desequilíbrio da microbiota vaginal, promovendo inflamação do epitélio cervical e proporcionando um epitélio suscetível a entrada de outros agentes, como o HPV. ${ }^{28}$

A maioria das lesões intraepiteliais cervicais é identificada entre os 20 e 30 anos de idade. A maioria dos laudos analisados no estudo pertenciam a pacientes entre 26-35 anos de idade com $31,01 \%$, seguida da faixa etária de 15-25 anos de idade, com 29,59\%. Trindade et al. ${ }^{24}$ também demonstraram a prevalência de mulheres jovens na adesão pelo exame, com mediana de idade de 35 anos. ${ }^{24}$

O elevado número de pacientes jovens no estudo, com diagnóstico de atipias ou lesões intraepiteliais cervicais, sugere que as orientações devem começar desde a adolescência, antes do início da vida sexual, 
evitando a transmissão do HPV e também de outras ISTs. Apesar da preconização do Ministério da Saúde que recomenda a realização do exame em mulheres a partir dos 25 anos de idade, as orientações sobre a realização do exame e a vacinação, que visam promoção e prevenção da saúde, devem ser implantadas desde a adolescência, proporcionando melhor qualidade de vida aos adolescentes e à sociedade. ${ }^{26}$

Fredrich e Renner ${ }^{28}$ observaram laudos de pacientes que foram atendidas pela rede pública, no estado do Rio Grande do Sul. A faixa etária que apresentou maior frequência de LSIL foi de 26-35 anos de idade e o diagnóstico de HSIL teve maior prevalência nas pacientes acima de 55 anos. ${ }^{28}$ Diante disso, analisamos que a gravidade das lesões, quando não diagnosticadas e não tratadas precocemente, pode evoluir com a idade das pacientes.

Freitas et al. ${ }^{29}$ também sugerem uma evolução das lesões com o aumento da idade, sendo que carcinoma escamoso invasivo e adenocarcinoma são mais frequentes acima dos 50 anos de idade. Os referidos autores relatam que lesões de alto grau surgem de lesões precursoras de baixo grau que poderiam ter sido diagnosticadas e tratadas anteriormente. $^{29}$

Portanto, é imprescindível adaptar os sistemas de saúde de acordo com as necessidades de cada comunidade, tanto na rede pública como na rede privada. Com a dispersão da população, devem ser elaborados programas que possam abranger um maior número de mulheres, buscando atender as necessidades que são encontradas em cada grupo. ${ }^{30}$ Devem ser desenvolvidos programas que exemplifiquem a importância do exame e do diagnóstico precoce das atipias e lesões que, quando não tratadas, poderão evoluir para o CCU.

A maneira mais eficaz de aumentar a conscientização sobre a realização do exame é a educação continuada. Sendo que as pacientes relatam ter conhecimento sobre a importância da realização do exame, porém, elas relatam que como não têm sintomas acreditam não ser necessária a procura por atendimento. ${ }^{31}$ Tornando-se essencial a realização de atividades educacionais com a população, pela equipe multidisciplinar de saúde, buscando promoção e prevenção de saúde das mulheres.

Com base nos dados analisados pelo presente estudo, evidencia-se que o percentual de lesões intraepiteliais cervicais é maior na rede pública em comparação com a rede privada, quando observadas às lesões de alto grau, o que indica um maior risco para o desenvolvimento do CCU em pacientes desta rede, visto que as lesões de alto grau possuem maior probabilidade de evolução para uma neoplasia. Essa diferença entre as duas redes de saúde pode ser explicada pelo tempo entre a realização do próximo exame, sendo que na rede pública é preconizado a realização após três anos, já na rede privada é realizado anualmente, e também a falta de orientações sobre a relevância da realização do exame.

Observa-se também que as pacientes acima dos 75 anos de idade apresentam maior prevalência de lesões de alto grau e de carcinoma, e ainda que as lesões de baixo grau apresentam em maior percentual pacientes jovens entre 15-25 e 26-35 anos de idade, sendo que a primeira faixa etária não é abrangida pela preconização da rede 
pública. O que ressalta a importância da realização do exame Preventivo do Câncer do Colo Uterino a partir do início da vida sexual, e não somente após os 25 anos de idade, a importância da vacinação e das orientações para a população, prevenindo assim o desenvolvimento de lesões e sua evolução.

A partir das conclusões obtidas, podem-se desenvolver atividades para a assistência continuada às mulheres, visando a promoção e prevenção de saúde.

\section{REFERÊNCIAS}

1. Carvalho MCMP, Queiroz ABA. Mulheres Portadoras de Lesões Precursoras do Câncer do Colo do Útero e HPV: Descrição do Perfil Socioeconômico e Demográfico. DST j. bras doenças sex. transm. 2011; 23 (1): 28-33.

2. Zardo GP, Fara FP, Mendes FG, Franco CAGS, Molina GVM, Melo GN, et al. Vacina como agente de imunização contra o HPV. Ciên Saúde Colet. 2014; 19 (9): 3799-808.

3. Agência Nacional de Vigilância Sanitária [Internet]. Registrada vacina do HPV contra 9 subtipos do vírus, 2017 [acesso em 07 abr 2019]. Disponível em: http://portal.anvisa.gov.br/rss/-/ asset_publisher/Zk4q6UQCj9Pn/content/id/3875990

4. Santos UM, Souza SEB. Papanicolau: Diagnóstico precoce ou prevenção do câncer cervical uterino. Rev. baiana saúde pública. 2013; 37 (4): 941-51.

5. Ministério da Saúde (BR). Diretrizes Brasileiras para o Rastreamento do Câncer do Colo do Útero. 2. ed. Rio de Janeiro: INCA; 2016.

6. Ministério da Saúde [Internet]. Câncer do colo do útero. Instituto Nacional De Câncer, 2018 [acesso em 28 mar 2019]. Disponível em: https://www.inca.gov.br/tipos-de-cancer/cancer-do-colo-do-utero

7. Fonseca AJ, Ferreira LP, Dalla-Benetta AC, Roldan CN, Ferreira MLS. Epidemiologia e impacto econômico do câncer de colo de útero no Estado de Roraima: a perspectiva do SUS. Rev. bras. ginecol. obstet. 2010; 32 (8): 386-92.

8. Dias MBK, Tomazelli JG, Assis M. Rastreamento do câncer de colo do útero no Brasil: análise de dados do Siscolo no período de 2002 a 2006. Epidemiol. serv. saúde. 2010; 19 (3): 293-306.

9. Nayar R, Wilbur DC. Sistema Bethesda Para Relato de Citologia Cervical: Definições, Critérios e Notas Explicativas. 3. ed. São Paulo: Livraria Livromed; 2018.

10. Rodrigues JF, Moreira BA, Alves TGS, Guimarães EAA. Rastreamento do Câncer do Colo do Útero na Região Ampliada Oeste de Minas Gerais, Brasil. Rev. enferm. Cent-Oeste Min. 2016; 6 (2): 2156-68.

11. Ministério da Saúde (BR). Nomenclatura Brasileira para Laudos Cervicais e Condutas Preconizadas: Recomendações para profissionais de saúde. 2. ed. Rio de Janeiro: Gdés; 2016.

12. Abdul-Karim FW, Powers CN, Berek JS, Sherman ME, Tabbara SO, Sidawy MK. Células escamosas atípicas. In: Nayar R, Wilbur DC. Sistema Bethesda Para Relato de Citologia Cervical: Definições, Critérios e Notas Explicativas. 3. ed. São Paulo: Livraria Livromed; 2018. p. 139-74.

13. Henry MR, Russell DK, Luff RD, Prey UM, Wright Jr TC, Nayar R. Anormalidade das células epiteliais: escamosas. In: Nayar R, Wilbur DC. Sistema Bethesda Para Relato de Citologia Cervical: Definições, Critérios e Notas Explicativas. 3. ed. São Paulo: Livraria Livromed; 2018. p. 175-238.

14. Fonseca FV, Tomasich FDS, Jung JE. Lesões cervicais intraepiteliais de alto grau: avaliação dos fatores determinantes de evolução desfavorável após conização. Rev. bras. ginecol. obstet. 2011; 33 (11): 334-40. 
15. Anjos SJSB, Vasconcelos CTM, Franco ES, Almeida PC, Pinheiro AKB. Fatores de risco para câncer de colo do útero segundo resultados de IVA, citologia e cervicografia. Rev. Esc. Enferm. USP. 2010; 44 (4): 912-20.

16. Borges MFSO, Dotto LMG, Koifman RJ, Cunha MA, Muniz PT. Prevalência do exame preventivo de câncer do colo do útero em Rio Branco, Acre, Brasil, e fatores associados à não-realização do exame. Cad. saúde pública. 2012; 28 (6): 1156-66.

17. Figueiredo MC, Melo Júnior JM, Segati KD. Prevalência de lesões precursoras para o câncer de colo do útero nas regiões do Brasil e sua relação com a cobertura do programa de rastreamento. Femina. 2014; 42 (6): 295-302.

18. Ministério da Saúde (BR). Inquérito Domiciliar sobre Comportamentos de Risco e Morbidade Referida de Doenças e Agravos não Transmissíveis: Brasil, 15 capitais e Distrito Federal 20022003. Rio de Janeiro: Gráfica Esdeva, 2004. p. 125.

19. Muller DK, Dias-da-Costa JS, Luz AMH, Olinto MTA. Cobertura do exame citopatológico do colo do útero na cidade de São Leopoldo, Rio Grande do Sul, Brasil. Cad. saúde pública. 2008; 24 (11): 2511-20.

20. Sousa AMV, Teixeira CCA, Medeiros SS, Nunes SJC, Salvador PTCO, Barros RMB, et al. Mortalidade por câncer do colo do útero no estado do Rio Grande do Norte, no período de 1996 a 2010: tendência temporal e projeções até 2030. Epidemiol. serv. saúde. 2016; 25 (2): 311-22.

21. Prática Clínica: Associamos ciência e tecnologia [Internet]. Cálculo amostral calculadora on-line [acesso em 10 maio 2019]. Disponível em: https://praticaclinica.com.br/anexos/ccolaborativa-calculo-amostral/ccolaborativa-calculo-amostral.php

22. Instituto Brasileiro de Geografia e Estatística [Internet]. Conheça Cidades e estados do Brasil, 2010 [acesso em 10 maio 2019]. Disponível em: https://cidades.ibge.gov.br/

23. Corrêa CSL, Lima AS, Leite ICG, Pereira LC, Nogueira MC, Duarte DAP, et al. Rastreamento do câncer do colo do útero em Minas Gerais: avaliação a partir de dados do Sistema de Informação do Câncer do Colo do Útero (SISCOLO). Cad saúde colet. 2017; 25 (3): 315-23.

24. Trindade GB, Maneti SA, Simões PW, Madeira K. Avaliação do rastreamento do câncer do colo do útero e sua periodicidade em um município de Santa Catarina. Medicina (Ribeirão Preto). 2017; 50 (1): 1-10.

25. Martins LTF, Fraga CDS, Andrade MS, Santos KJS, Paixão GPN, Bittencourt IS. Caracterização de mulheres com lesão pré-Maligna ou maligna no Exame Papanicolaou. Rev. enferm. UFPE. 2017; 11 (9): 3360-8.

26. Rocha CJ, Linassi C, Micheletti VD, Lora PS. Alterações celulares do HPV e de microflora de pacientes do SUS em São Leopoldo, RS Brasil. Enferm. foco. 2017; 8 (4): 26-30.

27. Costa RF, Barros SMO. Prevalência de lesões intraepiteliais em atipias de significado indeterminado em um serviço público de referência para neoplasias cervicais. Acta paul. enferm. 2011; 24 (3): 400-6.

28. Fredrich EK, Renner JDP. Alterações citopatológicas em exames de Papanicolaou na cidade de Santa Cruz do Sul, Rio Grande do Sul, Brasil. J. bras. patol. med. lab. 2019; 55 (3): 246-57.

29. Freitas RAP, Carvasan GAF, Morais SS, Zeferino LC. Prevalência das lesões neoplásicas do colo de útero: resultados de rastreamento citológico realizado em Campinas, São Paulo, Brasil. Rev. ciênc. méd. 2006; 15 (4): 307-14.

30. Fernandes NF, Galvão JR, Assis MMA, Almeida PF, Santos AM. Acesso ao exame citológico do colo do útero em região de saúde: mulheres invisíveis e corpos vulneráveis. Cad. saúde pública. 2019; 35 (10): 1-10.

31. Silva LSR, Cordeiro EL, Silva TM, Albuquerque AKDS, Ferreira MDR, Silva TLL. Adesão ao exame Papanicolau por mulheres jovens em unidade básica de saúde. Rev. enferm. UFPE. 2016; 10 (12): 4637-45. 


\section{Dados dos Autores}

\section{Dyenifer Portela de Lima}

Graduada em biomedicina pela Faculdade Especializada na Área de Saúde do Rio Grande do Sul (FASURGS), Passo Fundo/RS - Brasil. dyeniferporteladelima@gmail.com

\section{DENISE WOHLMEISTER}

Mestre em ciências farmacêuticas. Docente do curso de graduação em biomedicina na Faculdade Especializada na Área de Saúde do Rio Grande do Sul (FASURGS), Passo Fundo/RS - Brasil. prof. denisew@gmail.com

\section{Paula Wiethölter}

Doutora em fitotecnia. Docente na Faculdade Especializada na Área de Saúde do Rio Grande do Sul (FASURGS), Passo Fundo/RS - Brasil. paulawiet@gmail.com

Submetido em: 10-11-2020

Aceito em: 19-4-2021

\section{AGRADECIMENTOS}

Agradeço aos meus pais Ademar e Margarete pelo incentivo de sempre, ao meu irmão Luiz Felipe por tornar meus dias mais leves.

Agradeço à minha dinda e amiga Magali por estar sempre ao meu lado, pelo incentivo todos os dias.

Agradeço a todos os mestres que tive ao longo da graduação que, de alguma forma, contribuíram. 